\title{
mesure de la déformation radiale des éprouvettes de sol par un système de bagues
}

\author{
par \\ B. Félix \\ Ingénieur du Département des Sols et Fondations du Laboratoire central des Ponts et Chaussées
}

Pour interpréter les résultats de la plupart des essais effectués à l'aide d'une cellule triaxiale, la connaissance des déformations radiales de l'éprouvette est nécessaire. Dans un essai de cisaillement classique, par exemple, elles sont prises en compte pour le calcul de la section corrigée et donc de la contrainte normale $\sigma_{1}$ qui lui est appliquée. De la même manière, au cours d'un essai à contrainte imposée (essai de fluage par exemple), une correction est apportée à la charge verticale transmise par le piston, en fonction des déformations radiales. Plus généralement, la mesure de cette composante de la déformation est indispensable pour établir une loi de comportement, ne serait-ce qu'un coefficient de Poisson.

La cellule hermétiquement close dans laquelle est placée l'éprouvette rend malaisée l'installation d'instruments de mesure directe. Dans la pratique courante, les déformations radiales ne sont pas mesurées; elles sont calculées à partir des variations de volume et de hauteur. II existe cependant plusieurs techniques de mesures que nous allons présenter succinctement, avant de décrire plus en détail un nouvel appareillage qui offre un compromis intéressant entre trois qualités essentielles :

- le faible coût,

- la commodité d'emploi,

- la qualité de la mesure (précision, fidélité, exactitude).

\section{Détermination indirecte des déformations radiales}

Si un échantillon de forme constamment cylindrique a un volume initial $\mathrm{V}_{0}$ et une hauteur $\mathrm{H}_{0}$, sa déformation radiale est habituellement calculée par la formule (1) ou $\Delta \mathrm{V}$ est le volume drainé mesuré au volumètre et $\Delta \mathrm{H}$ la variation de hauteur assimilée au déplacement du piston.

$$
\varepsilon_{2}=\varepsilon_{3}=\sqrt{\frac{1+\frac{\Delta V}{V_{0}}}{1+\frac{\Delta H}{H}}}-1 .
$$

La variation de volume $\Delta \mathrm{V}$ est parfois mesurée par l'intermédiaire du fluide entrant dans la cellule. Cette technique, rarement utilisée, n'est intéressante que si I'on tient compte du gonflement de la cellule sous l'effet de la pression du fluide de confinement et des fuites inévitables qui se produisent le long du guidage du piston. Bishop et Lovenbury (1969), pour éviter ces inconvénients, ont eu l'idée d'immerger l'éprouvette dans une enceinte remplie de mercure dont le volume est mesuré. Le tout est placé dans une cellule triaxiale classique qui permet d'appliquer une pression de confinement. Cette solution présente deux inconvénients: l'échantillon est caché et une différence de pression latérale est introduite entre le haut et le bas de l'échantillon, étant donné la forte densité du mercure.

Le volume drainé hors de l'échantillon $\Delta V$ est égal à la variation de volume de l'éprouvette, si celle-ci est parfaitement saturée (naturellement ou par contrepression), et le volumètre est capable de la mesurer si l'étanchéité à l'eau et au gaz de la membrane qui entoure l'éprouvette est assurée, et si les tubulures de liaisons sont sans fuite, sont peu dilatables et ne contiennent pas de bulles de gaz. La sortie dans le volumètre d'une bulle qui occupait une longueur de quelques millimètres dans la tubulure, introduit une erreur qui peut être supérieure à $5 \%$ dans l'évaluation du volume réel de l'échantillon. (Robinet et al, 1978). Ces conditions sont d'autant moins respectées que l'essai triaxial dure plus longtemps.

\section{Méthodes directes de mesure}

\subsection{Mesure par visée optique}

Cette technique permet d'éviter l'implantation d'équi- 
pement à l'intérieur de la cellule. Escario et Uriel (1961) ont placé sur la surface extérieure de la cellule une série de lignes verticales gravées suivant les génératrices du cylindre et dans la partie supérieure une mire graduée en centimètres. Par alignement dans un même plan vertical, d'une ligne gravée extérieure, de l'image de la génératrice qui limite le profil de l'éprouvette et de celle des graduations de la mire, une lecture est effectuée. Le rayon de l'éprouvette dans une section horizontale quelconque est lié aux deux mesures correspondant aux deux génératrices limites de l'éprouvette par les lois de l'optique géométrique, dont les paramètres sont : les rayons intérieur et extérieur de la cellule et le rapport des indices de réfraction de l'eau et du matériau transparent constituant les parois de la cellule.

L'erreur due à l'excentrement éventuel de l'éprouvette peut être évitée en procédant par doubles mesures suivant deux lignes gravées extérieures qui sont diamétralement opposées. L'erreur absolue sur la mesure du diamètre est de l'ordre de $0,1 \mathrm{~mm}$, d'après les auteurs.

Blivet et Gestin (1974) ont déterminé le diamètre d'éprouvettes de craie par visée à l'aide d'une lunette placée à quelques mètres de la cellule. Le diamètre réel est déduit de la mesure grâce à un étalonnage.

\subsection{Mesure par ouverture d'un collier}

Ce type d'appareil a été proposé pour la première fois par Bishop et Henkel (1957), afin de mesurer les déformations radiales et le coefficient de pression des terres au repos $\mathrm{K}_{\mathrm{o}}$. Dans ce dernier cas, la pression de confinement $\sigma_{3}$ est asservie à la déformation radiale, de façon à maintenir celle-ci égale à zéro.

Le collier est formé de deux bras semi-circulaires pivotant autour d'une articulation. Le contact avec l'échantillon est assuré en deux points situés au milieu de chaque bras. Leur écartement entraîne l'ouverture du collier, qui est mesurée par un vernier (Blivet et Gestin, 1974) ou par un capteur de déplacement dont le signal est traité dans une chaîne d'enregistrement. Les deux points de contact ne restant pas diamétralement opposés par rapport à l'éprouvette, Robinet et al (1978) ont amélioré la forme et l'articulation des patins de palpage. L'erreur absolue sur la mesure du diamètre peut être inférieure au $1 / 100 \mathrm{~mm}$. Toutefois, le poids et l'encombrement de ce système de mesure rendent difficile l'installation de plusieurs exemplaires sur une même éprouvette. Pour ne pas perturber l'état de contrainte, le collier peut être supporté par un portique avec un système de contre-poids placé à l'intérieur de la cellule.

\subsection{Mesure par déformation d'un anneau élas- tique}

L'anneau est fabriqué à partir d'un ruban d'acier à haute limite d'élasticité. Il est fermé. Sa forme en plan comporte deux petits arcs de cercles qui assurent le contact avec l'éprouvette suivant deux zones diamétralement opposées. Ils sont reliés par deux lobes de forme circulaire (EI Rawayih, 1976) ou triangulaire arrondie (Vuaillat, 1980). Des jauges de déformation sont collées à l'intérieur et à l'extérieur de l'anneau, au milieu de chacune des parties lobées. Elles sont montées en pont complet, et convertissent en signal électrique la variation locale du rayon de courbure de l'anneau que provoque la déformation radiale de l'éprouvette. Ce système est léger et peu encombrant. Deux ou trois exemplaires peuvent être montés sur la même éprouvette. Son utilisation est surtout intéressante pour la mesure du coefficient $K_{0}$, mais les problèmes de collage des jauges et d'étalonnage rendent son utilisation difficile pour la mesure de déformations radiales importantes (supérieures à 2 pour cent environ).

\section{Un nouveau système de mesure par bagues coulissantes}

Les bagues, au nombre de deux, sont représentées développées sur la figure 1 et montées sur la figure 2 . Les deux modèles de bagues fabriqués enveloppent respectivement après cintrage, des cercles nominaux de $50,8 \mathrm{~mm}$ et de $76,2 \mathrm{~mm}$ de diamètre. Les bagues sont découpées dans un clinquant d'acier inoxydable de $0,2 \mathrm{~mm}$ d'épaisseur et recouvertes d'une fine pellicule de Téflon. La bague intérieure, de $17 \mathrm{~mm}$ de hauteur, est fendue; elle s'ouvre et se ferme suivant que le diamètre de l'éprouvette augmente ou diminue. Son rôle est de répartir la légère pression exercée par l'autre bague et surtout d'assurer, entre la languette graduée de celle-ci et la membrane qui entoure l'éprouvette, un jeu suffisant pour éviter un frottement important au droit de la fente (fig. 3). La bague de mesure est constituée d'une languette graduée qui s'enroule sur elle-même, en passant dans un guide situé à proximité du vernier qui sert à mesurer son ouverture avec une précision de $1 / 10 \mathrm{~mm}$. Un ressort de rappel dont le coefficient de raideur est égal à $7 \times 10^{-3} \mathrm{~N} / \mathrm{mm}$ environ, assure la fermeture des deux bagues et maintient ainsi le contact avec la surface extérieure de l'éprouvette. Ceci est d'autant plus facile que les bagues sont légères: $7,5 \mathrm{~g}$ pour la bague de diamètre nominal égal à $50,8 \mathrm{~mm}$ et $10 \mathrm{~g}$ pour celle de diamètre 76,2 . L'excès de pression latérale que les

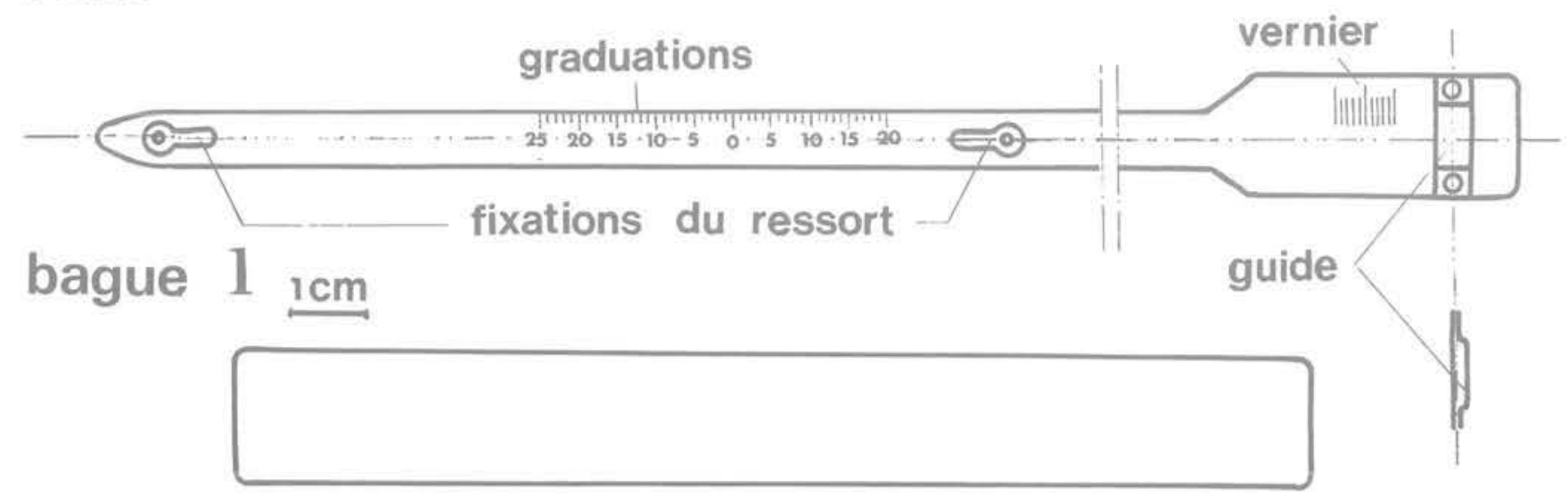

Fig. 1 Bagues 1 et 2 développées

bague 2 


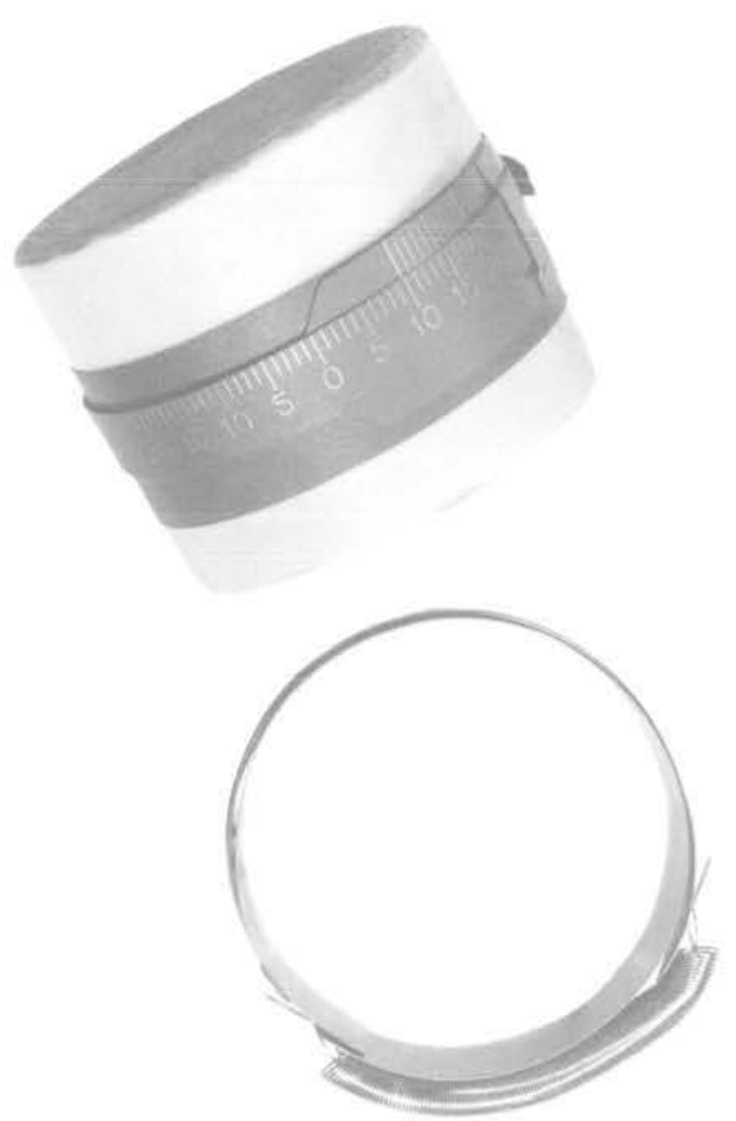

Fig. 2 Bague pour éprouvette de $50,8 \mathrm{~mm}$

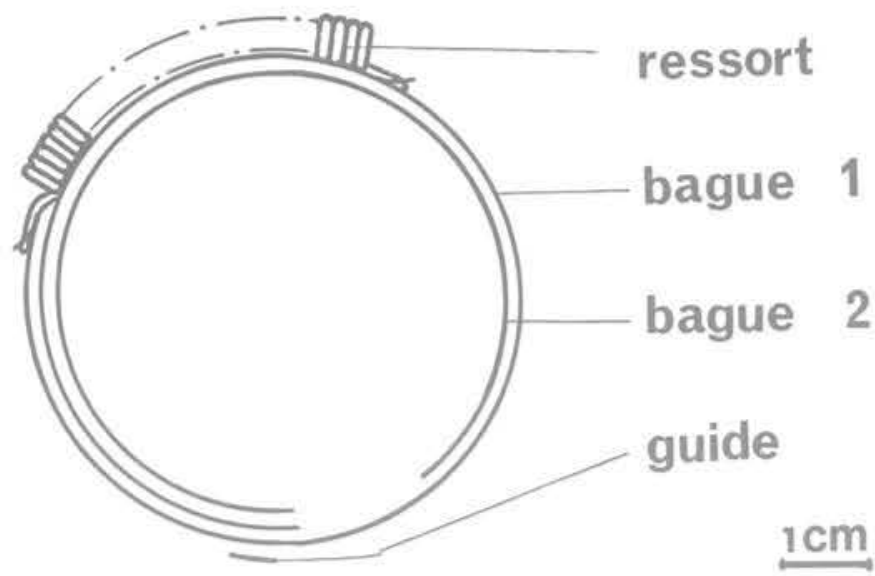

Fig. 3 Bagues 1 et 2 suivant l'axe de l'éprouvette

bagues exercent sur l'éprouvette a été mesuré en montant les bagues autour d'une membrane gonflable remplie d'air. La pression intérieure est représentée en fonction du diamètre interne de la bague, sur la figure 4, par un trait continu. Le trait pointillé correspond à la pression calculée à partir du coefficient de raideur du ressort et en supposant nuls les frottements des surfaces téflonées les unes sur les autres. La différence entre les deux courbes donne une idée de l'effet de la membrane et des frottements entre les bagues. La figure 5 montre 3 bagues montées sur une éprouvette de $50,8 \mathrm{~mm}$ de diamètre.
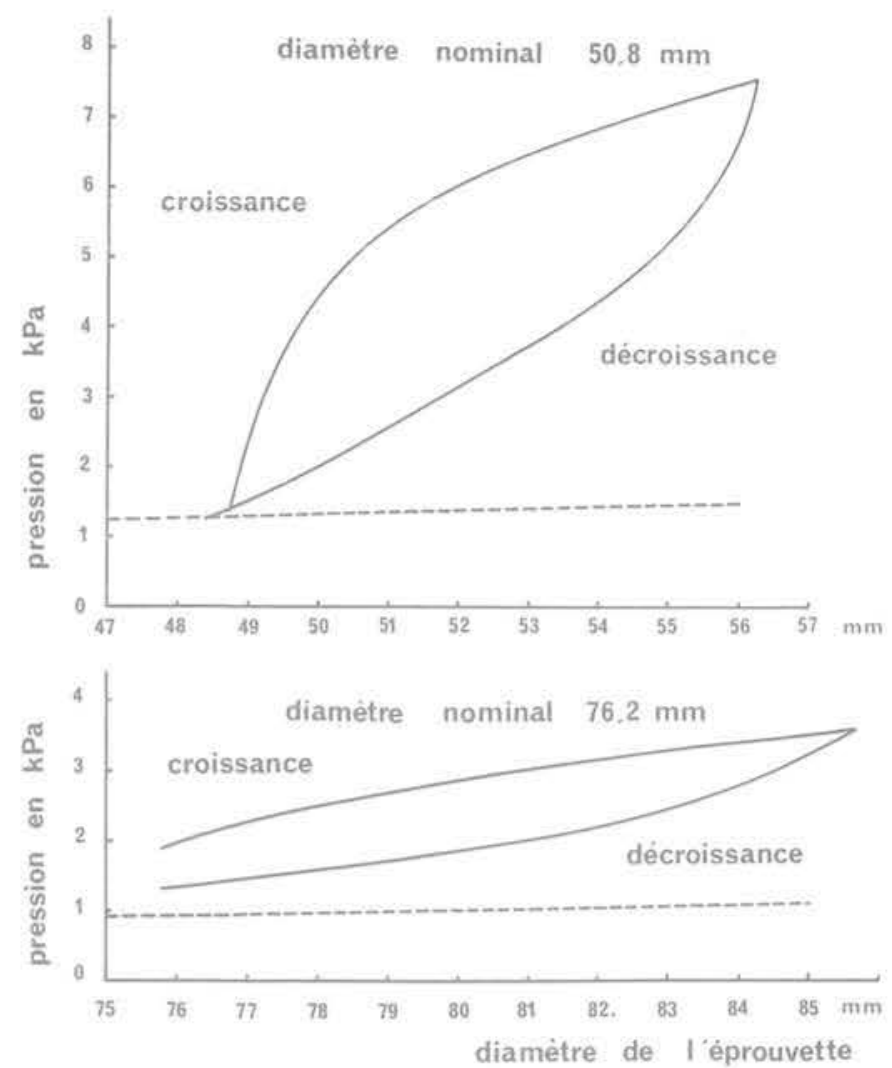

Fig. 4 Pression exercée sur l'éprouvette par la membrane et par la bague en fonction du diamètre mesuré (les pointillés représentent la pression due à la tension du ressort).

Fig. 5 Bagues montées sur une éprouvette

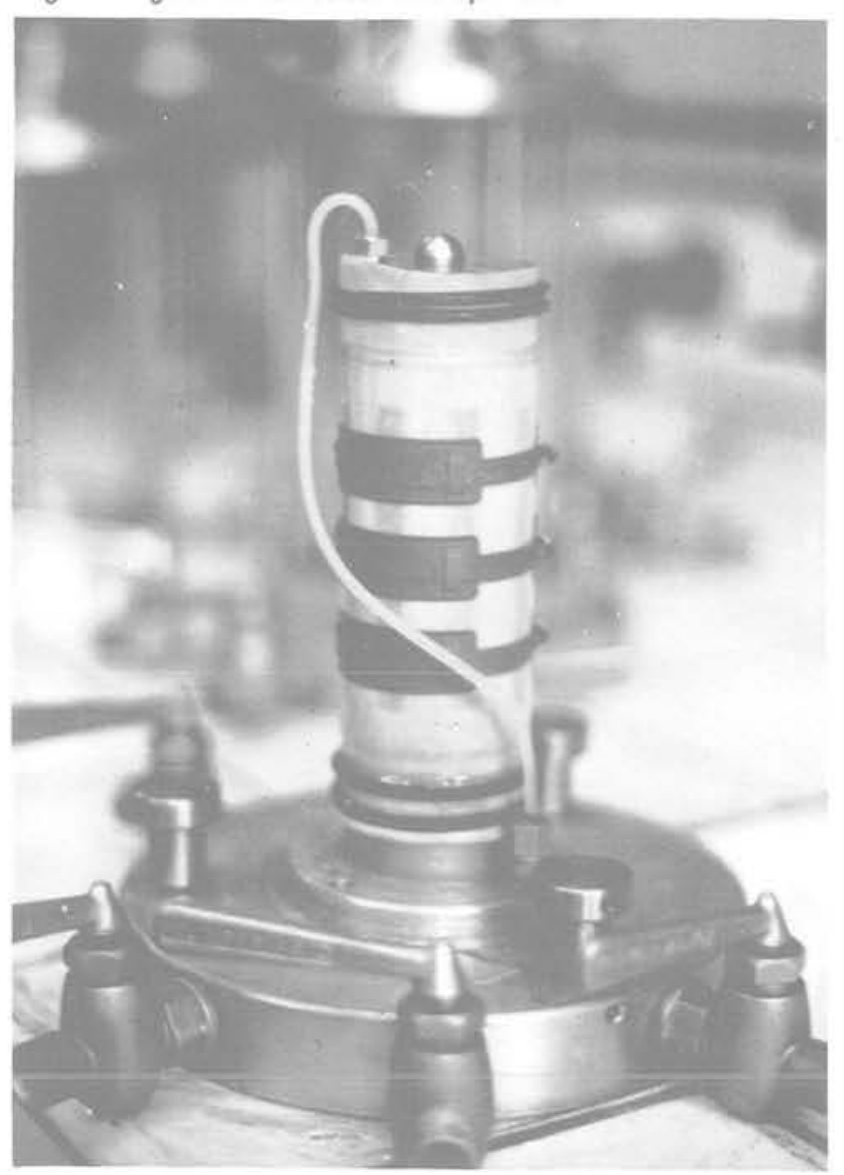


La lecture sur le vernier de l'ouverture de la bague est effectuée à travers la paroi de plexiglass de la cellule et le liquide de remplissage. L'effet de grossissement dô à la différence entre les indices de réfraction de l'air et des autres milieux facilite la lecture qui est mise en relation, par un étalonnage, avec le diamètre du cylindre enveloppé. Chaque bague, dont la forme après fabrication n'est pas parfaitement circulaire et l'est encore moins après déformation, a été placée sur une série de 4 cylindres de duralumin usiné à la tolérance $\pm 0,01 \mathrm{~mm}$. On obtient ainsi quatre relations entre la mesure du vernier et le diamètre du cylindre et, par extrapolation linéaire, un tableau de correspondance. Les bagues sont conçues pour mesurer, avec une erreur absolue inférieure à $3 / 100 \mathrm{~mm}$, un diamètre d'éprouvette qui augmente ou qui diminue (au cours d'une consolidation par exemple). Les déformations maximales tolérées excèdent $\pm 8 \%$.

\section{Quelques résultats obtenus}

Les bagues ont été utilisées pour une importante série d'essais, réalisée en particulier sur une argile molle prélevée à Cubzac-les-Ponts et une argile très raide (argile des Flandres de Winezeele). Sur chaque éprouvette ont été placées trois bagues à intervalles réguliers, égaux au quart de sa hauteur totale.

La figure 6 montre l'évolution, au cours d'une consolidation isotrope sous $80 \mathrm{kPa}$, de la section longitudinale d'une éprouvette d'argile de Cubzac-lesPonts drainée par sa base et par sa surface latérale, recouverte de papier filtre. Les déplacements des points du contour, qui sont multipliés par 20 , montrent que l'éprouvette en cours de consolidation, prend une forme approximativement tronconique.

Fig. 7 Consolidation isotrope sous $80 \mathrm{kPa}$, comparaison des déformations volumiques calculées et mesurées (durée de l'essai : 35 jours)

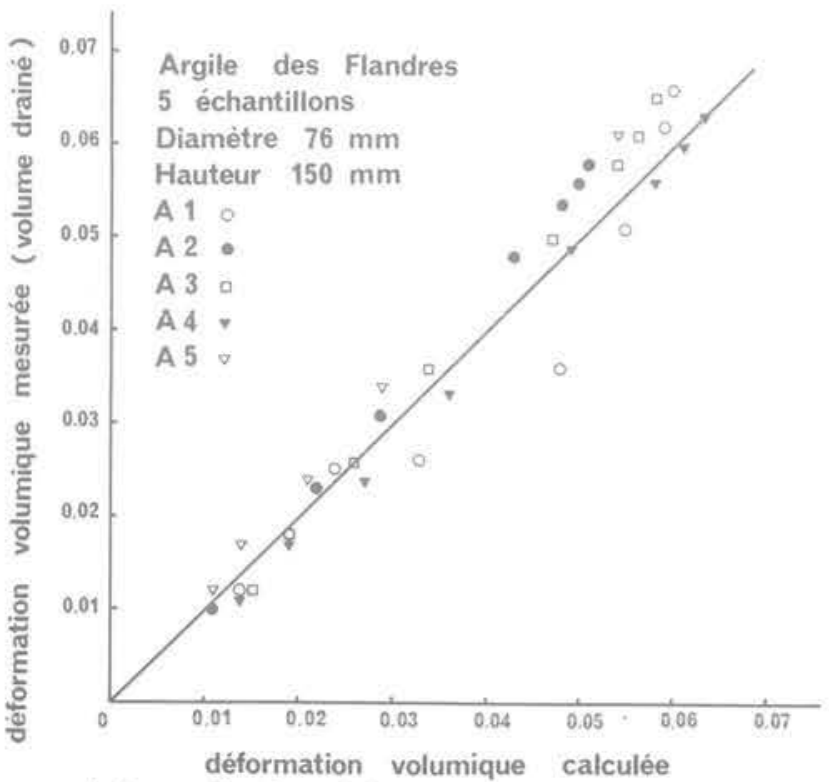

( à partir des déformations radiales et verticales )

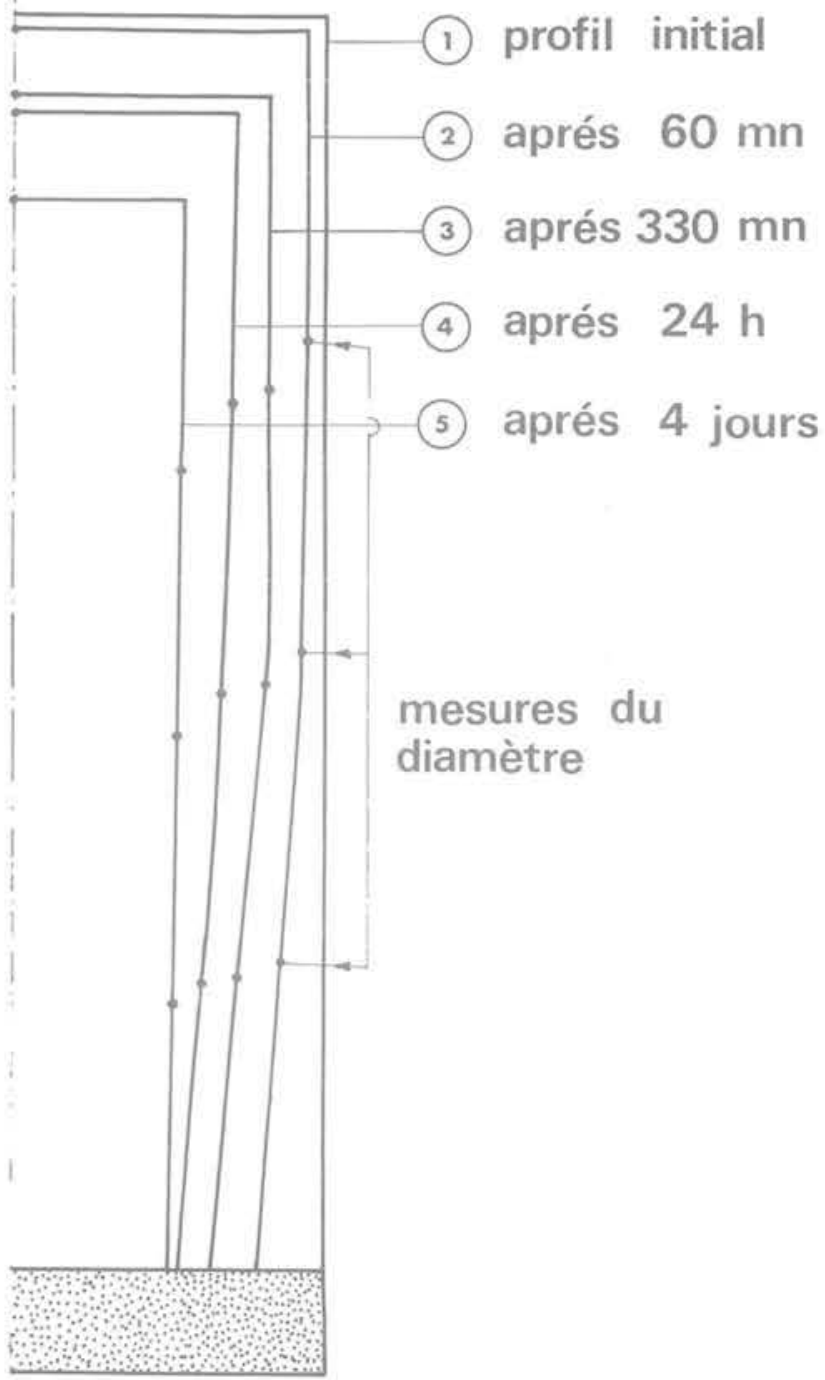

Fig. 6 Evolution du profil d'une éprouvette d'argile de Cubzac-les-Ponts au cours de la consolidation (les déplacements du contour sont multipliés par 20)

Fig. 8 Consolidation isotrope sous $500 \mathrm{kPa}$, comparaison des déformations volumiques calculées et mesurées (durée de l'essai : 7 jours)

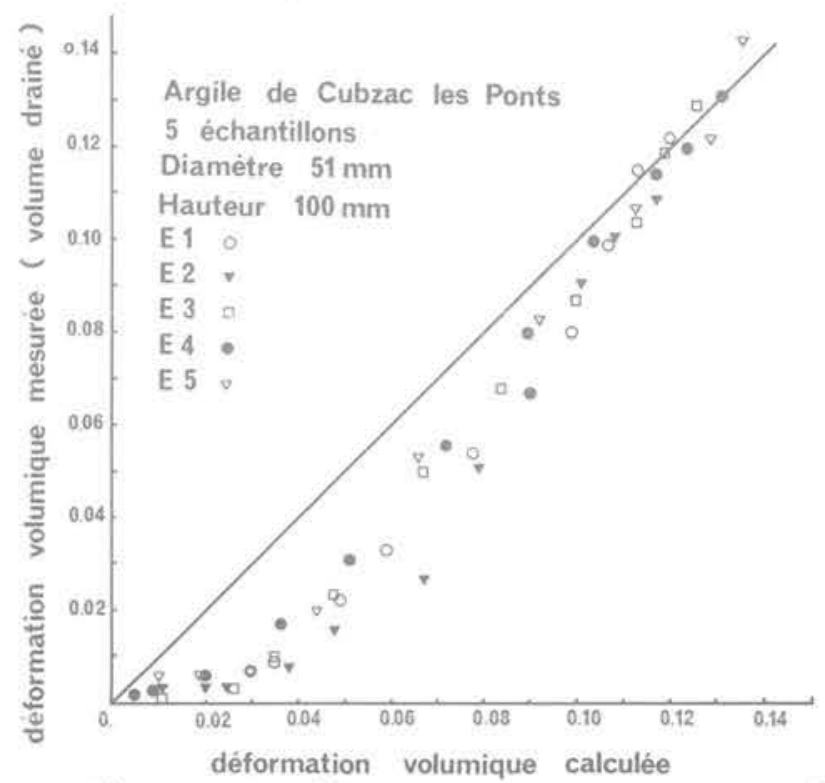

( à partir des déformations radiales et verticales ) 
5 éprouvettes ont subi une consolidation de ce type. Le volume de liquide drainé, mesuré au volumètre, est égal, après 35 jours, à $12 \%$ environ du volume initial des éprouvettes. Les différentes valeurs de la déformation volumique mesurées au volumètre sont reportées sur l'axe des ordonnées de la figure 7 , tandis que la variation de volume calculée à partir des variations de la hauteur et du diamètre figure sur l'axe des abscisses. On remarque que les premières mesures effectuées au volumètre dans les 4 jours qui suivent l'application de la charge, sont nettement inférieures à la variation de volume calculée à partir des mesures linéaires. Par contre, à la fin de la consolidation, les résultats présentent des discordances minimes, inférieures à $10 \%$. Le phénomène est dû à la présence de gaz dans l'argile de Cubzac-les-Ponts dont le degré de saturation est inférieur à $96 \%$. Aucune contre-pression n'étant appliquée, ce gaz occupe initialement une fraction importante du volume, qui diminue après l'application de la pression $\sigma_{3}$ de confinement. L'éprouvette de sol subit une déformation volumique, sans pour autant perdre de liquide interstitiel par drainage. Lorsque les surpressions interstitielles sont dissipées (après 4 jours), le gaz occupe à nouveau la même fraction du volume total. La perte de volume de l'éprouvette depuis le début de l'essai est alors comparable au volume drainé et les déformations volumiques déterminées par les deux méthodes ont tendance à concorder.

La consolidation isotrope sous $500 \mathrm{kPa}$ de 5 éprouvettes de $76,2 \mathrm{~mm}$ de diamètre d'argile des Flandres parfaitement saturée suit à cet égard une évolution très différente. La figure 8 , dont la présentation est analogue à celle de la précédente, montre que, dans ce cas, les déformations calculées et mesurées concordent d'un bout à l'autre de l'essai. Après 7 jours, le volumètre indique des déformations qui s'échelonnent de 5,8 à $6,5 \%$ et qui ont tendance à être légèrement supérieures à celle calculées à partir des déformations linéaires. On peut y voir une conséquence des défauts d'étanchéité des joints et de la membrane qui enveloppe l'éprouvette.

\section{Conclusion}

La déformation radiale des éprouvettes est habituellement déterminée à partir du volume de liquide drainé, mesuré par le volumètre, et de la variation de hauteur de l'éprouvette. Cette méthode peut conduire à de grosses erreurs, en particulier lorsque l'éprouvette contient du gaz. De nombreuses méthodes de mesure directe du diamètre ont été mise au point, mais aucune n'a reçu jusqu'ici de large utilisation. Les mesures optiques évitent l'installation d'appareillage à l'intérieur de la cellule, mais l'erreur absolue est importante.
Par contre, les mesures par ouverture d'un collier ou déformation d'un anneau élastique sont très précises. Elles sont effectuées généralement par l'intermédiaire d'un système électronique d'acquisition des données. Le poids des colliers, l'étalonnage du système et son instabilité dans le temps peuvent constituer des inconvénients. La mesure porte à chaque fois sur un seul diamètre par section et se trouve à la merci du changement de forme de cette dernière. Le système de bagues proposé a été conçu pour être simple, facile à mettre en œuvre et peu coûteux $(200 \mathrm{~F}$ environ en 1978). II suit l'évolution du périmètre des éprouvettes de sol soumises à des essais triaxiaux, et fournit une indication précise du diamètre moyen avec une erreur absolue égale à $3 / 100$ de millimètre. La perturbation apportée aux contraintes appliquées est minime, quelles que soit les déformations de l'éprouvette et le système fonctionne pour des variations importantes positives ou négatives du diamètre (pour des déformations de l'ordre de $\pm 8 \%$ ).

\section{Références bibliographiques}

Bishop A.W., Henkel D.J. (1962). «Chap. II Principal features of the triaxial apparatus" et "Appendix 6 Developpements in the Period 1957-61 „. The Measurement of Soil Properties in the Triaxial Test, $2^{\circ}$ édition. Edward Arnold (Publishers) LTD, 1962, p. 33-82 et p. 180-212.

Bishop A.W., Lovenbury H.T. (1969). «Creep characteristics of two undisturbed clays s, août 1969, Compte rendu de la $7^{\circ}$ Conférence Internationale de MS et de TF, Mexico, vol. 1, p. 29-38.

Blivet J. C., Gestin F. (1974). «Rhéologie de la craie remaniée. Mesure des relations effort-déformation ". bull. de liaison des Laboratoires des Ponts et Chaussées, $n^{\circ} 73$, septembre-octobre 1974, p. 28-35.

El-Ruwayih A.A. (1976), «Design manufacture and performance of a lateral strain device ", Geotechnique, vol. $26, n^{\circ} 1$, mars 1976 , p. 215-216.

Escario V., Uriel S. (1961). "Optical Methods of Measuring the Cross Section of Samples in the triaxial Test $n$, juillet 1961, Compte rendu de la $5^{\circ}$ Conférence Internationale de MS et de TF, Paris, vol. 1, p. 89-93.

Robinet J. C. et al. (1978). «Système de mesure de la déformation radiale dans un essai biaxial ", bull. de liaison des Laboratoires des Ponts et Chaussées, $n^{\circ} 93$, janvier-février 1978, p. 134-137.

Vuaillat P. (1980). «Propriétés visqueuses d'une argile: Expérience et formulation incrémentalen. Thèse de docteur-ingénieur, présentée à l'Université de Grenoble, soutenue le 21 février 1980. 
